\title{
Application of Stability Theory in Study of Local Dynamics of Nonlinear Systems
}

\author{
Tahmineh Azizi, Gabriel Kerr \\ Department of Mathematics, Kansas State University, Manhattan, KS, USA \\ Email: tazizi@ksu.edu,gdkerr@math.ksu.edu
}

How to cite this paper: Azizi, T. and Kerr, G. (2020) Application of Stability Theory in Study of Local Dynamics of Nonlinear Systems. Journal of Applied Mathematics and Physics, 8, 1180-1192.

https://doi.org/10.4236/jamp.2020.86089

Received: May 15, 2019

Accepted: June 20, 2020

Published: June 23, 2020

Copyright (C) 2020 by author(s) and Scientific Research Publishing Inc. This work is licensed under the Creative Commons Attribution International License (CC BY 4.0).

http://creativecommons.org/licenses/by/4.0/

\section{(c) (i) Open Access}

\begin{abstract}
Investigating local dynamics of equilibrium points of nonlinear systems plays an important role in studying the behavior of dynamical systems. There are many different definitions for stable and unstable solutions in the literature. The main goal to develop stability definitions is exploring the responses or output of a system to perturbation as time approaches infinity. Due to the wide range of application of local dynamical system theory in physics, biology, economics and social science, it still attracts many researchers to play with its definitions to find out the answers for their questions. In this paper, we start with a brief review over continuous time dynamical systems modeling and then we bring useful examples to the playground. We study the local dynamics of some interesting systems and we show the local stable behavior of the system around its critical points. Moreover, we look at local dynamical behavior of famous dynamical systems, Hénon-Heiles system, Duffing oscillator and Van der Pol equation and analyze them. Finally, we discuss about the chaotic behavior of Hamiltonian systems using two different and new examples.
\end{abstract}

\section{Keywords}

Local Dynamics, Hénon-Heiles System, Duffing Oscillator, The Van Der Pol Equation, Hamiltonian Function

\section{Introduction}

A dynamical system describes the evolution of a system over time using a set of mathematical laws. Also, it can be used to predict the interactions between different components of a system [1] [2]. There are two main methods to model the dynamical behaviors of a system, continuous time modeling, discrete-time mod- 
eling [1] [2] [3]. When the time between two measurements is negligible, the continuous time modeling governs the evolution of the system, however, when there is a gap between two measurements, discrete-time system modeling comes to play. Ordinary differential equations are the tool to model a continuous system and iterated maps represent the discrete generations [4] [5].

In this paper we will be concerned with continuous dynamical systems which are defined by differential equations. Indeed, some famous examples of dynamical systems can be written in terms of differential equations: the harmonic oscillator, the pendulum and double pendulum, or the N-body problem [4]-[9]. A dynamical system is a triple $\left(M, \Phi_{t}, K\right)$ where $M$ is called the phase space and is usually a smooth manifold or a subset of $\mathbb{R}^{n}, \Phi_{t}: M \times K \rightarrow M$, called the evolution, is a smooth action of $K$ in $M$ and $K$ is either a subset of $\mathbb{R}$ in the case of a continuous time dynamical system or a subset of $\mathbb{Z}$ in the case of a discrete time dynamical system. The smooth action $\Phi_{t}(x)$ describes the evolution with time $t \in K$ of a point $x$ in the phase space $M$ [4] [5] [6] [7].

Stability of a system is one of the most important parts of the studying the dynamical behavior of a system. Generally speaking, an unstable and also a chaotic system are not useful and we like to work with a system with stable and or periodic behavior (although chaos is a known behavior for many systems and sometimes people look for different strategies for chaotification of a system for different purposes [10] [11] [12]).

There are different definitions for stability, however, all they have this common fact that a system is stable if perturbation, external input and or intentionally applied signals cannot make the system get away from the equilibrium point [13] [14]. There are three possibilities for dynamical behavior of a system after applying a perturbation [5]:

1) The system state would return to the equilibrium state.

2) The system state would not return to the equilibrium state but stays near to that state.

3) The system state diverges from the equilibrium state.

Mathematically speaking, the equilibrium state $x^{*}$ is stable if for each initial conditions $x(0)$ close enough to $x^{*}$, the corresponding trajectory $x(t)$ remains near $x^{*}$ for all $t \geq 0$.

$$
\forall \varepsilon>0 \quad \exists \delta>0 \quad:\left\|x(0)-x^{*}\right\|<\delta \Rightarrow\left\|x(t)-x^{*}\right\|<\varepsilon, \quad \forall t \geq 0
$$

In this paper, we present some results regarding the study of local dynamics of non-linear continuous time dynamical systems. We provide different examples to display stable and unstable limit cycles and we demonstrate the numerical results for each case. Also, we study the local dynamics of three well known physical systems, Henon-Heiles system, Duffing oscillator and Van der pol equations and we display the evolution of solutions of the system in time. Finally, we discuss about the chaos in Hamiltonian systems and we provide two examples to show chaos in Hamiltonian systems. 


\section{Dynamical Systems Playground}

When we start to analyze the local dynamics of non-linear systems, the first step is finding the critical points and then exploring how the trajectories of the system evolving in the neighborhood of critical point. This analysis helps us to find out how other solutions or trajectories of the system behave when they get close to the critical points. Another step to analyze a dynamical system is studying the trajectories which trace out a limit cycle or a closed curve. In this case, the solution $x(t)$ of the system will go around and create a closed curve $C$ with a certain period $T$. Therefore, the solutions $x(t)=(x(t), y(t))$ of the system when it becomes periodic change to be $x(t+T)=x(t), y(t+T)=y(t)$ for all $t$. Any trajectories which are close to the limit cycle $C$, follow the same behavior as the limit cycle $C$. For instance, they can get spiral in toward $C$, or they can spiral away from $C$, which demonstrates if the closed curve $C$ is stable or unstable. See Figure 1.

The root point for $F_{1}(X, Y)=\left(-X^{2}-Y^{2}\right) \exp \left(-X^{2}-Y^{2}\right)$ is $(X, Y)=(0,0)$. The Taylor expansion for $F_{1}(X, Y)$ at $(X, Y)=(0,0)$ has the following form:

$$
T_{n}(X, Y)=-Y^{2} \mathrm{e}^{-Y^{2}}+X^{2} \mathrm{e}^{-Y^{2}}\left(Y^{2}-1\right)-\frac{1}{2} X^{4} \mathrm{e}^{-Y^{2}}\left(Y^{2}-2\right)+O\left(X^{5}\right)
$$

As it is clear from Figure 1, the point $(0,0)$ is a stable fixed point and also the maximum of $F_{1}(X, Y)$ occurs at $(X, Y)=(0,0)$ and it is:

$$
\max \left\{\left(-X^{2}-Y^{2}\right) \exp \left(-X^{2}-Y^{2}\right)\right\}=0
$$

In and Figure 2, we see $F_{2}(X, Y)=\left(X^{2}+Y^{2}\right) \exp \left(-X^{2}-Y^{2}\right)$. The root for $F_{2}(X, Y)$ is $(X, Y)=(0,0)$. The Taylor expansion for $F_{2}(X, Y)$ at $(X, Y)=(0,0)$ has the following form:

$$
T_{n}(X, Y)=Y^{2} \mathrm{e}^{-Y^{2}}-X^{2} \mathrm{e}^{-Y^{2}}\left(Y^{2}-1\right)+\frac{1}{2} X^{4} \mathrm{e}^{-Y^{2}}\left(Y^{2}-2\right)+O\left(X^{5}\right)
$$

The minimum of $F_{2}(X, Y)$ happens for $(X, Y)=(0,0)$ which is equal:

$$
\min \left\{\left(X^{2}+Y^{2}\right) \exp \left(-X^{2}-Y^{2}\right)\right\}=0
$$

For $F_{2}(X, Y)$ the point $(X, Y)=(0,0)$ is unstable.

Another example, $F_{3}(X, Y)=X \exp \left(-X^{2}-Y^{2}\right)$ which has been displayed in Figure 3. The maximum of $F_{3}(X, Y)$ occurs at $(X, Y)=(-1,0)$ and $(X, Y)=(1,0)$ and it equals to:

$$
\max \left\{X^{2} \exp \left(-X^{2}-Y^{2}\right)\right\}=\frac{1}{\mathrm{e}}
$$

Also, the Taylor expansion for $F_{2}(X, Y)$ at $X=0$ has the following form:

$$
T_{n}(X, Y)=X^{2} \mathrm{e}^{-Y^{2}}-X^{4} \mathrm{e}^{-Y^{2}}+\frac{1}{2} X^{6} \mathrm{e}^{-Y^{2}}-\frac{1}{6} X^{8} \mathrm{e}^{-Y^{2}}+O\left(X^{9}\right)
$$

As we can see in Figure 3, $(X, Y)=(-1,0)$ and $(X, Y)=(1,0)$ are stable.

In Figure 4, we can see for $F_{4}(X, Y)=Y^{2} \exp \left(-X^{2}-Y^{2}\right),(X, Y)=(0,-1)$ and $(X, Y)=(0,1)$ are stable. 


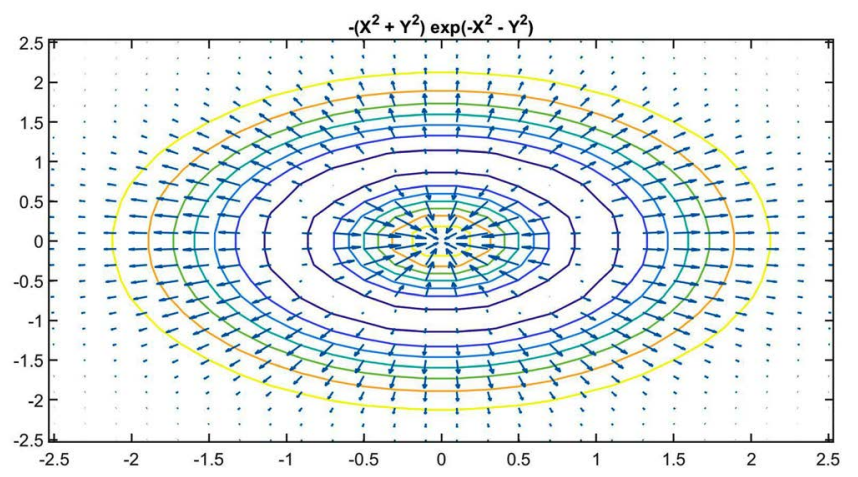

Figure 1. $F_{1}(X, Y)=\left(-X^{2}-Y^{2}\right) \exp \left(-X^{2}-Y^{2}\right)$, local dynamics.

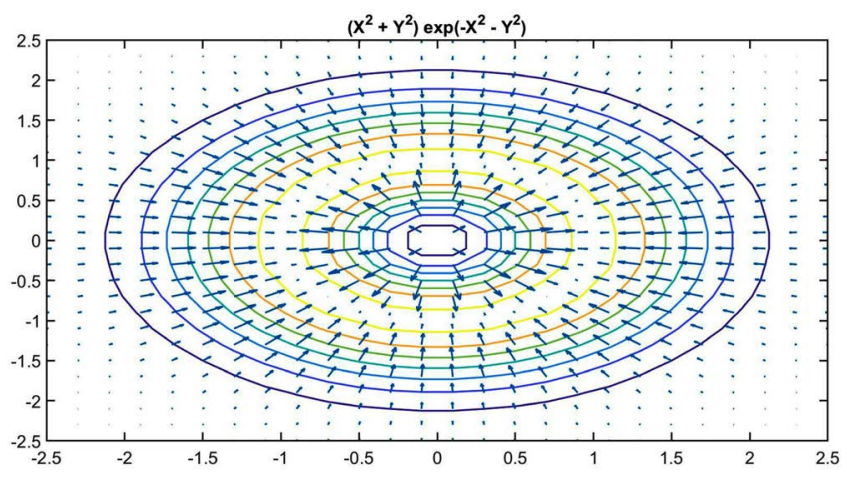

Figure 2. $F_{2}(X, Y)=\left(X^{2}+Y^{2}\right) \exp \left(-X^{2}-Y^{2}\right)$, local dynamics.

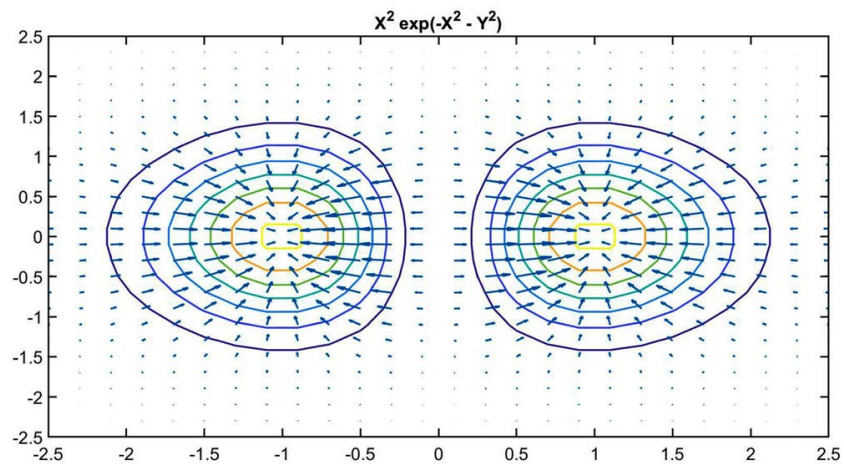

Figure 3. $F_{3}(X, Y)=X \exp \left(-X^{2}-Y^{2}\right)$, local dynamics.

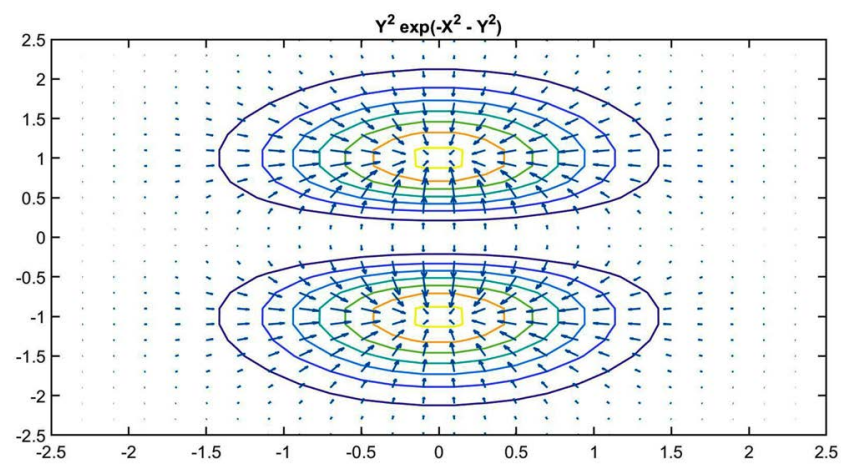

Figure 4. $Z=Y^{2} \exp \left(-X^{2}-Y^{2}\right)$, local dynamics. 
The maximum of $F_{4}(X, Y)$ occurs at $(X, Y)=(0,-1)$ and $(X, Y)=(0,1)$ and equals to

$$
\max \left\{Y^{2} \exp \left(-X^{2}-Y^{2}\right)\right\}=\frac{1}{\mathrm{e}}
$$

Also, the Taylor expansion for $F_{4}(X, Y)$ at $X=0$ has the form

$$
T_{n}(X, Y)=Y^{2} \mathrm{e}^{-Y^{2}}-X^{2} \mathrm{e}^{-Y^{2}} Y^{2}+\frac{1}{2} X^{4} \mathrm{e}^{-Y^{2}} Y^{2}+O\left(X^{5}\right)
$$

For $F_{5}(X, Y)=\left(X^{2} Y^{2}\right) \exp \left(-X^{2}-Y^{2}\right)$ (Figure 5), $(X, Y)=(0,0)$ is a root and Taylor expansion for $F_{5}(X, Y)$ at $X=0$ has the form

$$
T_{n}(X, Y)=X^{2} \mathrm{e}^{-Y^{2}} Y^{2}-X^{4} \mathrm{e}^{-Y^{2}} Y^{2}+\frac{1}{2} X^{6} \mathrm{e}^{-Y^{2}} Y^{2}-\frac{1}{6} X^{8} \mathrm{e}^{-Y^{2}} Y^{2}+O\left(X^{9}\right)
$$

here, $(X, Y)=(-1,-1)$ and $(X, Y)=(-1,1)$ give the maximum of $F_{5}(X, Y)$ which is

$$
\max \left\{X^{2} Y^{2} \exp \left(-X^{2}-Y^{2}\right)\right\}=\frac{1}{\mathrm{e}^{2}}
$$

Finally, in Figure 6, $F_{6}(X, Y)=-\left(X^{2} Y^{2}\right) \exp \left(-X^{2}-Y^{2}\right)$ has a root at $(X, Y)=(0,0)$ and Taylor expansion for $F_{6}(X, Y)$ at $X=0$ has the form

$$
T_{n}(X, Y)=-X^{2} \mathrm{e}^{-Y^{2}} Y^{2}+X^{4} \mathrm{e}^{-Y^{2}} Y^{2}-\frac{1}{2} X^{6} \mathrm{e}^{-Y^{2}} Y^{2}+\frac{1}{6} X^{8} \mathrm{e}^{-Y^{2}} Y^{2}+O\left(X^{9}\right)
$$

here, $(X, Y)=(-1,-1)$ and $(X, Y)=(-1,1)$ give the minimum of $F_{6}(X, Y)$ which is

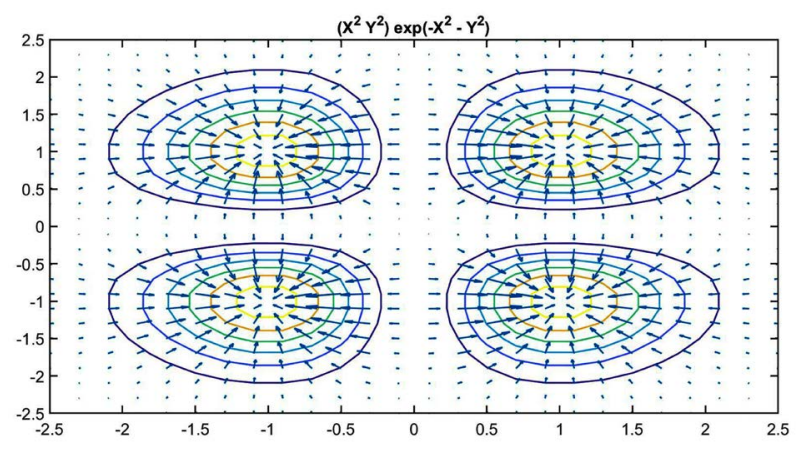

Figure 5. $F_{5}(X, Y)=\left(X^{2} Y^{2}\right) \exp \left(-X^{2}-Y^{2}\right)$, local dynamics.

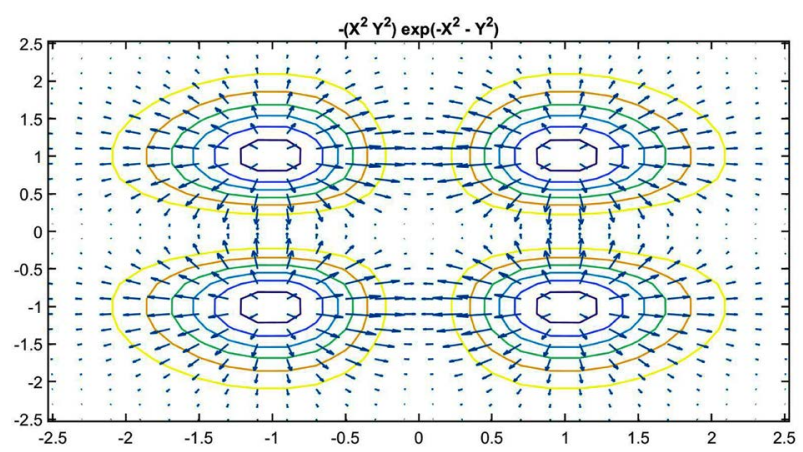

Figure 6. $F_{6}(X, Y)=-\left(X^{2} Y^{2}\right) \exp \left(-X^{2}-Y^{2}\right)$, local dynamics. 


$$
\min \left\{-X^{2} Y^{2} \exp \left(-X^{2}-Y^{2}\right)\right\}=-\frac{1}{\mathrm{e}^{2}}
$$

\section{Application of Continuous Dynamical Systems Modeling}

\subsection{Hénon-Heiles System}

The Hénon-Heiles potential is one of the simplest examples of classical mechanics and Hamiltonian systems [15] [16] [17] [18]. The Hénon-Heiles Hamiltonian demonstrates the emotion of stars around a galactic center. In 1964, Michael Hénon and Carl Heiles simplified the problem of the emotion of stars around a galactic center by using a Hamiltonian to describe the motion of stars near the equilibrium [16]. The Hénon-Heiles system has a vide application is studying chaotic dynamics in a system. If the energy of the motion becomes close to the bounding energy of the potential sink which is surrounding the center of the potential, this system displays chaotic dynamics feit 1984 wave.

Consider the following nonlinear system of ordinary differential equations

$$
\left\{\begin{array}{l}
\frac{\mathrm{d} x}{\mathrm{~d} t}=y \\
\frac{\mathrm{d} y}{\mathrm{~d} t}=x-x^{2}
\end{array}\right.
$$

The Hamiltonian function for this system has the form

$$
H(x, y)=\frac{y^{2}}{2}-\frac{x^{2}}{2}+\frac{x^{3}}{2}
$$

For any $x, y$ satisfying (13), we have $\frac{\mathrm{d} H}{\mathrm{~d} t}=0$. For any solution $(x(t), y(t))$ of system (13), the Hamiltonian $H(x(t), y(t))$ is constant, it means $\frac{\mathrm{d}}{\mathrm{d} t} H(x(t), y(t))=0$. This is a very nice property of Hamiltonian function which is a conserved quantity for a system of ordinary differential equations and it is constant along all solution curves of the system.

The solution curves are given by $H(x, y)=C$. Here, there are two non degenerate critical points $(0,0)$ and $(-1,0)$. The critical point $(0,0)$ is a saddle point and the eigenvectors corresponding to this critical points are $(1,-1)^{\mathrm{T}}$ and $(1,1)^{\mathrm{T}}$. The critical point $(-1,0)$ is a center. Figure 7 displays the level curves or contours of four different Hamiltonian functions.

Consider the following Hamiltonian functions

$$
\begin{aligned}
& H_{1}(x, y)=-\frac{y^{2}}{2}+\frac{x^{2}}{2}+\frac{x^{3}}{2} \\
& H_{2}(x, y)=\frac{y^{2}}{2}-\frac{x^{2}}{2}+\frac{x^{3}}{2} \\
& H_{3}(x, y)=\frac{y^{2}}{2}+\frac{x^{2}}{2}+\frac{x^{3}}{2} \\
& H_{4}(x, y)=\frac{y^{2}}{2}+\frac{x^{2}}{2}-\frac{x^{3}}{2}
\end{aligned}
$$



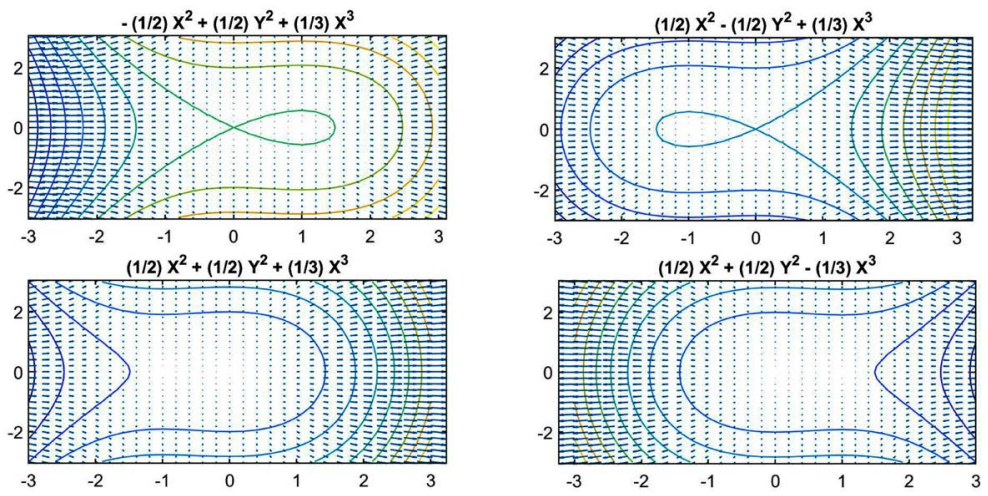

Figure 7. Hénon-Heiles system phase portraits.

These Hamiltonian functions (15)-(18), are corresponding to different system of ordinary differential equations. The Hamiltonian function $H_{1}(x, y)$ has a critical point at $(1,0)$ and the Hamiltonian function $H_{2}(x, y)$ has a critical point at $(-1,0)$. As we can see in Figure 7 , the stable and unstable manifolds from the origin for $H_{1}(x, y)$ and $H_{2}(x, y)$ form a homoclinic orbit which we can not see this property in Hamiltonian functions $H_{3}(x, y)$ and $H_{4}(x, y)$. This homoclinic loop connects the critical point $(0,0)$ to itself and it takes infinite amount of time to make connection. For Hamiltonian functions $H_{1}(x, y)$ and $H_{2}(x, y)$, the critical point $(0,0)$ is called a saddle-node equilibrium and the Jacbian matrix of the system has a zero eigenvalues at this equilibrium point. However, the critical point $(0,0)$ for the Hamiltonian functions $H_{3}(x, y)$ and $H_{4}(x, y)$ demonstrates another kind of dynamics and it is called the Bogdanov-Takens equilibrium point and the Jaobian matrix in this case has two zero eigenvalues. As it can be seen in Figure 7, the critical point $(0,0)$ is unstable which is the property of Bogdanov-Takens equilibrium point.

\subsection{Duffing Oscillator}

The Duffing oscillator is a single ordinary differential equation which represents a nonlinear damped driven oscillator. This simple nonlinear system displays different kinds of dynamical behaviors from periodic and regular behaviors to chaos. When we add a driving force and friction, we can see this simple equation exhibit chaotic behavior [19] [20] [21]. The Duffing oscillator equation has the following form

$$
y^{\prime \prime}+\alpha y^{\prime}+\beta y+\gamma x^{3}=0, \quad y(0)=A, y^{\prime}(0)=B
$$

The law of energy conservation mentions that this is impossible to see chaotic motion in a single degree of freedom. Therefore, with adding a driving force and damping, the energy conservation would be eliminated. Then, the equations of motion has the form

$$
\left\{\begin{array}{l}
\frac{\mathrm{d} y(1)}{\mathrm{d} t}=y(2) \\
\frac{\mathrm{d} y(2)}{\mathrm{d} t}=-b y(2)-\alpha y(1)-\beta y^{3}(1)+a m p \sin (w t)
\end{array}\right.
$$


We have demonstrated different dynamical behaviors of (20) in Figures 8-10. The Duffing oscillator can be used to model different physical phenomenon such as stiffening springs, beam buckling, nonlinear electronic circuits, superconducting Josephson parametric amplifiers, and ionization waves in plasmas [22].
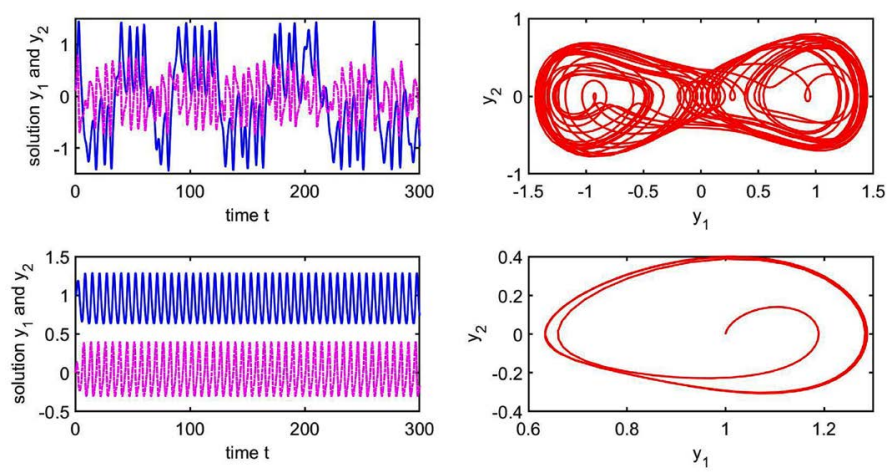

Figure 8. Chaotic solutions of Duffing oscillator (20) for $a m p=0.42$, $b=0.5, \quad \alpha=-1.0, \quad \beta=1.0, w=1.0$, Periodic solutions of Duffing oscillator (20) for $a m p=0.35, b=0.75, \alpha=-1.0, \beta=1.0, w=1.0$.
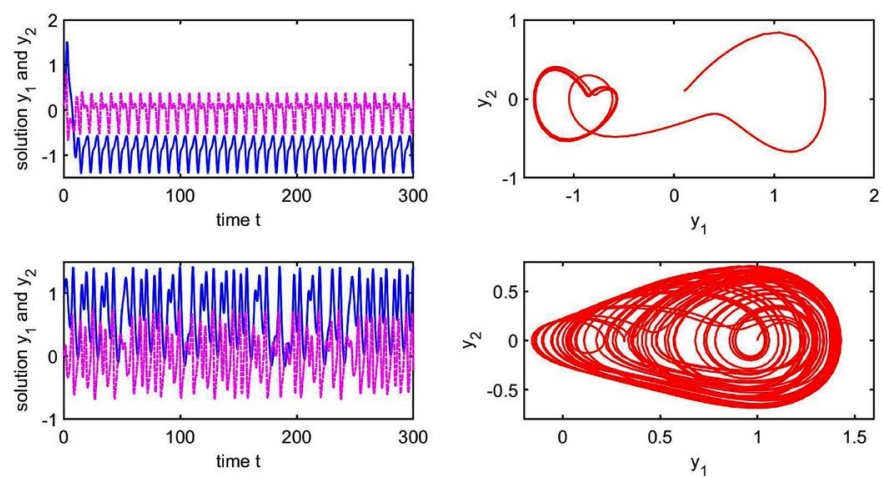

Figure 9. Periodic solutions of Duffing oscillator (20) for $a m p=0.45$, $b=0.45, \alpha=-1.0, \beta=1.0, w=0.75$, Chaotic solutions of Duffing oscillator (20) for $a m p=0.4, b=0.49, \alpha=-1.0, \quad \beta=1.0, w=1.1$.
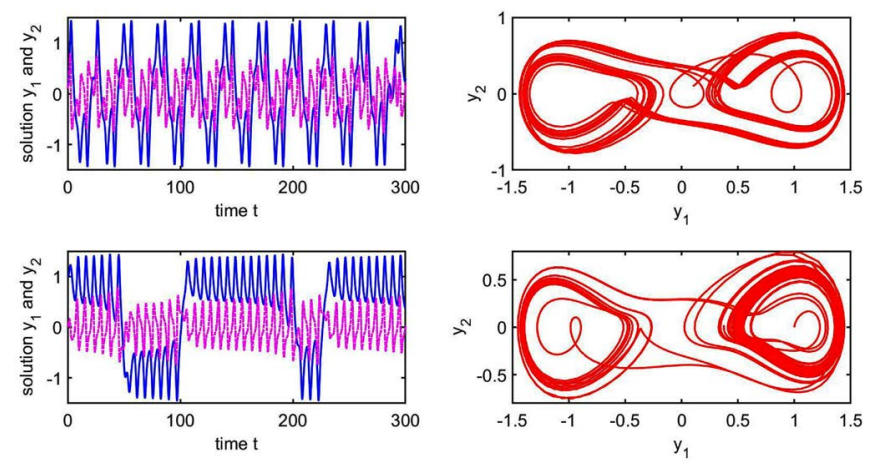

Figure 10. Periodic solutions of Duffing oscillator (20) for $a m p=0.43$, $b=0.51, \alpha=-1.0, \quad \beta=1.0, w=1.05$, Chaotic solutions of Duffing oscillator (20) for $a m p=0.39, b=0.47, \alpha=-1.0, \beta=1.0, w=0.9$. 


\subsection{The Van Der Pol Equation}

Van der Pol equation which is a well known second order ordinary differential equation with cubic nonlinearity has attracted many researchers in different field of science. This self oscillatory system, Van der Pol oscillator, has been considered as very useful mathematical model for many complicated systems [23] [24] [25]. Mathematical representation of the Van der Pol system has the form

$$
x^{\prime \prime}+\mu\left(x^{2}-1\right) x^{\prime}+x=0
$$

where constant $\mu$ is a positive parameter depending on the tube constants. This equation represents describes the current $x(t)$ in a certain type of vacuum tube. We can write (21) as a first order system of differential equations:

$$
\left\{\begin{array}{l}
\frac{\mathrm{d} x}{\mathrm{~d} t}=y \\
\frac{\mathrm{d} y}{\mathrm{~d} t}=-\mu\left(x^{2}-1\right) x^{\prime}-x
\end{array}\right.
$$

The numerical integration of Equation (22) has been represented in Figures 11-14.

As we can see, depending on different values for $\mu$, solutions look like periodic motion. When $\mu$ small values, this motion is nearly sinusoidal, however for larger values of $\mu$, the solutions seem to be relaxation oscillations which means solutions are similar to a series of step functions and jump twice per cycle between the positive and the negative values.

\section{Chaos in Continuous Dynamical Systems}

In this section, we assume that there is a Hamiltonian function with two degrees of freedom and it is given by $H=H_{0}+\varepsilon H_{1}$. Here, we consider $\varepsilon$ to be a very small parameter, $H_{0}$ an integrable Hamiltonian system and $H_{1}$ makes $H$ to be non-integrable. For $\varepsilon=0$ and also for $0<\varepsilon \ll 1$, there exist quasi periodic cycles which are known as KAM tori. However, under perturbation, these quasi periodic cycles will be deformed and KAM tori will be dissolved gradually as we increase $\varepsilon$. This phenomenon can be observed in Figure 15 and Figure 16.
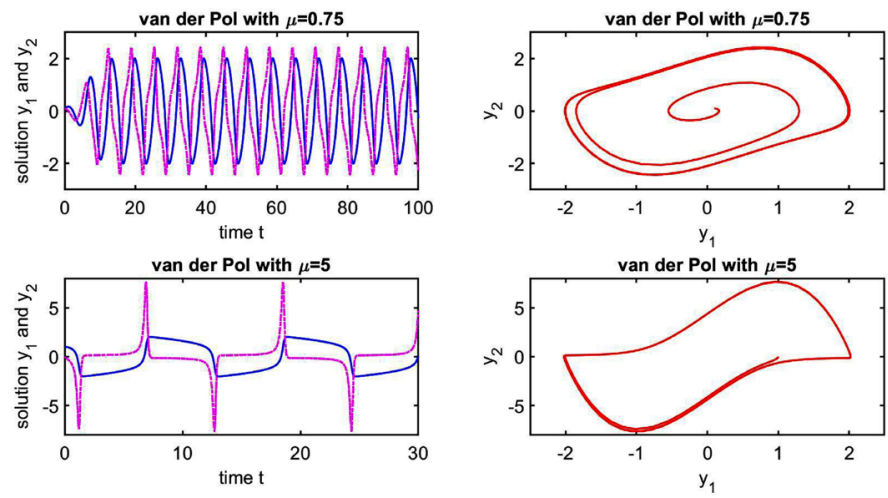

Figure 11. Solutions of Van der Pol Equation (22) with $\mu=0.75$ and $\mu=5$. 

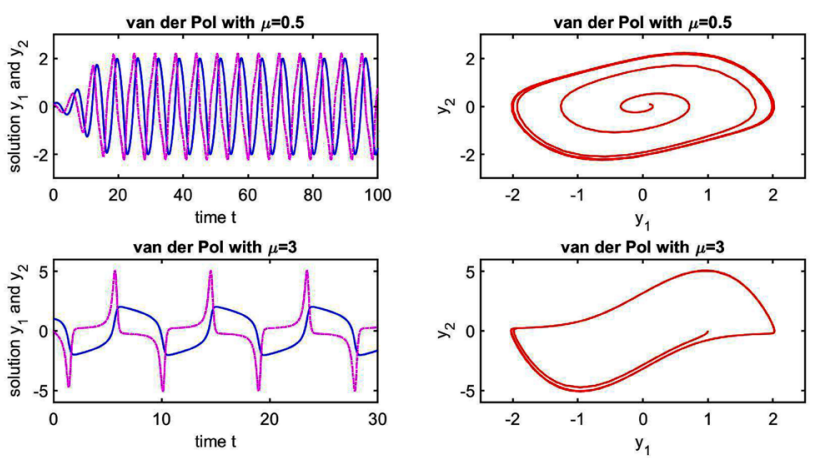

Figure 12. Solutions of Van der Pol Equation (22) with $\mu=0.5$ and $\mu=3$.
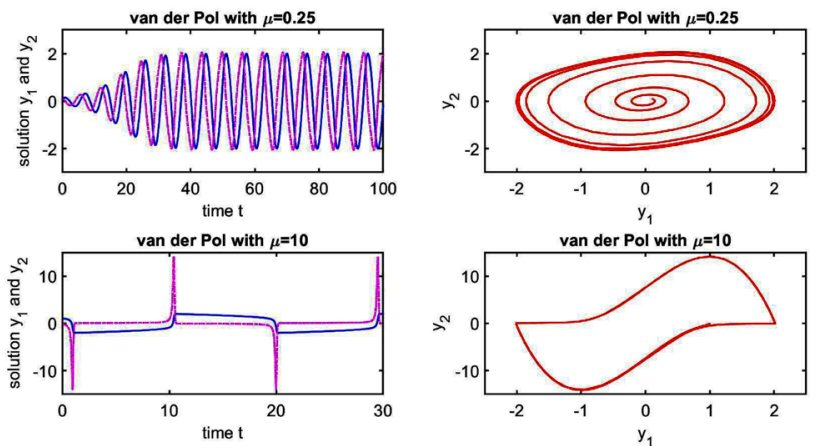

Figure 13. Solutions of Van der Pol Equation (22) with $\mu=0.25$ and $\mu=10$.
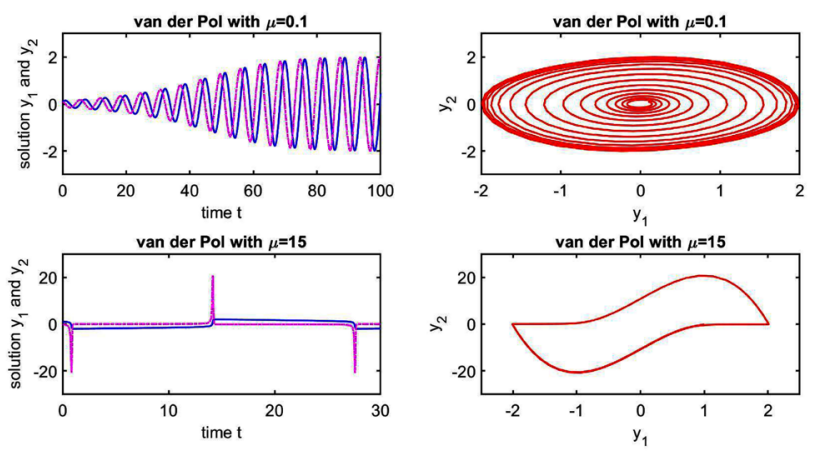

Figure 14. Solutions of Van der Pol Equation (22) with $\mu=0.1$ and $\mu=15$.

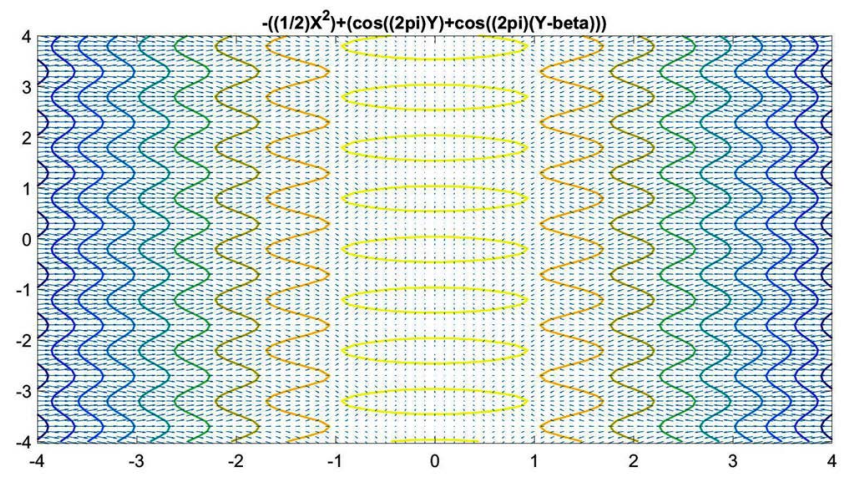

Figure 15. Dissolving the KAM tori caused by perturbation, $H_{1}(x, y)=-\left((1 / 2) x^{2}\right)+\varepsilon(\sin ((\pi / 2) y)+\sin ((\pi / 2)(y-\beta)))$. 


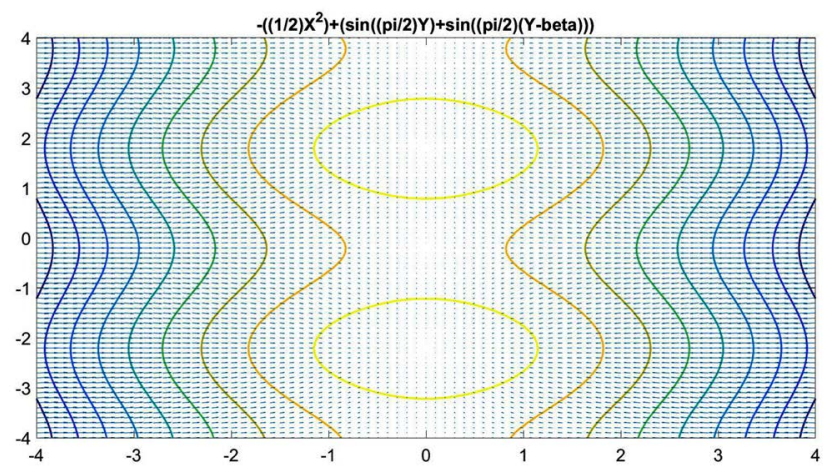

Figure 16. Dissolving the KAM tori caused by perturbation,

$$
H_{2}(x, y)=-\left((1 / 2) x^{2}\right)+\varepsilon(\cos ((2 \pi) y)+\cos ((2 \pi)(y-\beta))) \text {. }
$$

According to, KAM theory when $x$ is irrational, then the torus is preserved for small perturbation $\varepsilon$. But, proportional tori and adjacent irrational tori would be destroyed. Also, the stable manifold and unstable manifold of the saddle point which are intersecting transversely, appear to be Smale horseshoe and chaotic motion. As $\varepsilon$ increases gradually, these chaotic layers grow and they envelope larger area in phase space [26] [27].

\section{Conclusion}

Dynamic systems modeling have been frequently used to describe different physical systems and have a very important role in predicting the interactions between multiple components of a system over time. In the present study, we explored different dynamical behaviors of some continuous dynamical systems, from stable and regular motions to periodic and limit cycles, and then chaotic and irregular oscillations. We started with studying the local dynamics of some vector fields and we demonstrated the local stable behavior of the system around its critical points. We continued this paper with studying the well known problems which have been used a lot for different physical purposes. Hénon-Heiles system, Duffing oscillator and Van der Pol equation are three important dynamical examples which have been widely studied numerically. We demonstrated the stable and unstable manifolds from the origin form a homoclinic orbit in Hénon-Heiles system and we discussed about the local dynamical behaviors of its critical points. We showed that the critical point $(0,0)$ is a saddle point and critical point $(-1,0)$ is a center. For Duffing oscillator, which can be used to model different physical phenomenon, we showed the periodic and chaotic motions of the system using time series. Also, for Van der Pol equation, we presented the limit cycle solutions and periodic behavior of the system. We concluded that depending on different values for $\mu$, solutions look like periodic motion. When $\mu$ small values, this motion is nearly sinusoidal, however for larger values of $\mu$, the solutions seem to be relaxation oscillations, which means solutions are similar to a series of step functions and jump twice per cycle between the positive and the negative values. Finally, we discussed about the 
chaos in Hamiltonian systems and we provided two interesting and different examples which exhibit chaotic behaviors. We assume a Hamiltonian function with two degrees of freedom and it can be obtained by adding an integrable Hamiltonian system and a non-integrable Hamiltonian system. We showed that for $\varepsilon=0$ and also for $0<\varepsilon \ll 1$, there exist quasi periodic cycles which are known as KAM tori. However, under perturbation, these quasi periodic cycles will be deformed and KAM tori will be dissolved gradually as we increase $\varepsilon$.

\section{Conflicts of Interest}

The authors declare no conflicts of interest regarding the publication of this paper.

\section{References}

[1] Strogatz, S. (2001) Nonlinear Dynamics and Chaos: With Applications to Physics, Biology, Chemistry, and Engineering (Studies in Nonlinearity). KRC Press, Boca Raton.

[2] Strogatz, S.H. (2018) Nonlinear Dynamics and Chaos with Student Solutions Manual: With Applications to Physics, Biology, Chemistry, and Engineering. CRC Press, Boca Raton. https://doi.org/10.1201/9780429399640

[3] Rozhdestvensky, K., Ryzhov, V., Fedorova, T., Safronov, K., Tryaskin, N., Sulaiman, S.A., Ovinis, M. and Hassan, S. (2020) Computer Modeling and Simulation of Dynamic Systems Using Wolfram System Modeler. Springer, Berlin.

[4] Guckenheimer, J. and Holmes, P. (2013) Nonlinear Oscillations, Dynamical Systems, and Bifurcations of Vector Fields. Springer Science \& Business Media, Berlin, 42.

[5] Wiggins, S. (2003) Introduction to Applied Nonlinear Dynamical Systems and Chaos. Springer Science \& Business Media, Berlin, 2.

[6] LaSalle, J.P. (1976) The Stability of Dynamical Systems. SIAM, Philadelphia, 25. https://doi.org/10.21236/ADA031020

[7] Shub, M. (2013) Global Stability of Dynamical Systems. Springer Science \& Business Media, Berlin.

[8] Michel, A.N., Hou, L. and Liu, D.R. (2008) Stability of Dynamical Systems. Springer, Berlin.

[9] Kuznetsov, Y.A. (2013) Elements of Applied Bifurcation Theory. Springer Science \& Business Media, Berlin, 112.

[10] Zhang, H.G., Liu, D.R. and Wang, Z.L. (2009) Controlling Chaos: Suppression, Synchronization and Chaotification. Springer Science \& Business Media, Berlin.

[11] Wang, X.F. and Chen, G.R. (2000) Chaotification via Arbitrarily Small Feedback Controls: Theory, Method, and Applications. International Journal of Bifurcation and Chaos, 10, 549-570. https://doi.org/10.1142/S0218127400000372

[12] Chen, H.-K., Lin, T.-N. and Chen, J.-H. (2005) Dynamic Analysis, Controlling Chaos and Chaotification of a SMIB Power System. Chaos, Solitons \& Fractals, 24, 1307-1315. https://doi.org/10.1016/j.chaos.2004.09.081

[13] Liu, Y. and Anderson, B.D.O. (1989) Singular Perturbation Approximation of Balanced Systems. International Journal of Control, 50, 1379-1405.

https://doi.org/10.1080/00207178908953437 
[14] Yedavalli, R.K. (1985) Perturbation Bounds for Robust Stability in Linear State Space Models. International Journal of Control, 42, 1507-1517. https://doi.org/10.1080/00207178508933441

[15] Feit, M.D. and Fleck Jr., J.A. (1984) Wave Packet Dynamics and Chaos in the Hénon-Heiles System. The Journal of Chemical Physics, 80, 2578-2584. https://doi.org/10.1063/1.447051

[16] Ozaki, J. and Kurosaki, S. (1996) Periodic Orbits of Hénon Heiles Hamiltonian: Bifurcation Phenomenon. Progress of Theoretical Physics, 95, 519-529. https://doi.org/10.1143/PTP.95.519

[17] Zotos, E.E. (2015) Classifying Orbits in the Classical Hénon-Heiles Hamiltonian System. Nonlinear Dynamics, 79, 1665-1677. https://doi.org/10.1007/s11071-014-1766-6

[18] Zotos, E.E. (2017) An Overview of the Escape Dynamics in the Hénon-Heiles Hamiltonian System. Meccanica, 52, 2615-2630. https://doi.org/10.1007/s11012-017-0647-8

[19] Kovacic, I. and Brennan, M.J. (2011) The Duffing Equation: Nonlinear Oscillators and Their Behaviour. John Wiley \& Sons, Hoboken. https://doi.org/10.1002/9780470977859

[20] Wiggins, S. (1987) Chaos in the Quasiperiodically Forced Duffing Oscillator. Physics Letters A, 124, 138-142. https://doi.org/10.1016/0375-9601(87)90240-4

[21] Nayfeh, A.H. and Sanchez, N.E. (1989) Bifurcations in a Forced Softening Duffing Oscillator. International Journal of Non-Linear Mechanics, 24, 483-497. https://doi.org/10.1016/0020-7462(89)90014-0

[22] Korsch, H.J., Jodl, H.-J. and Hartmann, T. (2007) Chaos: A Program Collection for the PC. Springer Science \& Business Media, Berlin.

[23] Tsatsos, M. (2006) Theoretical and Numerical Study of the Van der Pol Equation. Doctoral Dissertation, Aristotle University of Thessaloniki, Thessaloniki.

[24] Guckenheimer, J. (1980) Dynamics of the van der Pol Equation. IEEE Transactions on Circuits and Systems, 27, 983-989. https://doi.org/10.1109/TCS.1980.1084738

[25] Guckenheimer, J., Hoffman, K. and Weckesser, W. (2003) The Forced van der Pol Equation I: The Slow Flow and Its Bifurcations. SIAM Journal on Applied Dynamical Systems, 2, 1-35. https://doi.org/10.1137/S1111111102404738

[26] Zaslavsky, G.M. and Zaslavskij, G.M. (2005) Hamiltonian Chaos and Fractional Dynamics. Oxford University Press on Demand, Oxford.

[27] Combescure, M. (2005) Hamiltonian Chaos and Fractional Dynamics. Journal of Physics A, Mathematical and General, 38, 5380. https://doi.org/10.1088/0305-4470/38/23/B02 